\title{
ORAL KAPOSI'S SARCOMA AND THE GLOBAL HHV-8 STORY
}

Newell W. Johnson, David J. Speicher

Griffith Health Institute, Griffi th University, Qld, Australia

Background: Kaposi's sarcoma (KS) remains the major AIDS-defining neoplasm worldwide, although incidence has declined in HIV-populations with widespread antiretroviral treatment. In high-incidence regions, oral lesions are common, accurate and rapid diagnosis being important for patient care. We sought to improve methods for diagnosis and characterisation of virus in patients from Australia and Africa.

Methods: Histopathology and immunocytochemistry (ICC) for LANA-1 HHV-8 antigen on paraffin sections; HHV-8 ORF73 and ORF26 qPCR on DNA from lesions.

Results: In Australian patients $(\mathrm{n}=37)$, KS lesions from various body sites had characteristic histomorphology: all were positive by ICC. HHV-8 involvement was confirmed by rtPCR, and a qPCR assay developed.1 Sequencing showed most to be Subtype-A with two being Subtype-C. From Kenya, 17 of 19 non-oral, but only 15 of 28 presumed oral-KS, were confirmed by ICC: there were seven pyogenic granulomas, two deep mycoses and four HHV8 negative inflamed/ulcerated mucosae. All Kenyan samples produced the same HHV-8 Subtype, thought to be because of laboratory contamination.

Conclusions: ICC for HHV-8 LANA-1 antigen is an essential tool for accurate diagnosis, especially for differential diagnosis of common oral lesions. PCR is best avoided in resourcepoor settings unless strict laboratory hygiene can be guaranteed.

\section{Reference}

1. Speicher DJ, Johnson NW. Detection of human herpesvirus 8 by quantitative polymerase chain reaction: development and standardisation of methods. BMC Infect Dis 2012; 12: 210. 